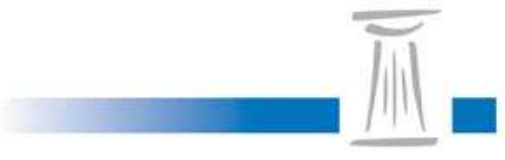

MISSION DE RECHERCHE

DROIT \& JUSTICE

APPEL A PROJETS

$\diamond$ Projets à faire parvenir en :

\section{0 exemplaires}

$\diamond$ Date limite de réception des projets :

\section{JUIN 2009}

Cachet de la poste faisant foi ou dépôt à la Mission avant 17 heures

$\diamond$ Durée maximale de la recherche :

\section{4 mois}

\section{Mission de recherche Droit et Justice}

30, rue du Château des Rentiers 75013 Paris

Téléphone : 01.44.77.66.60

Télécopie : 01.44.77.66.70

Courriel :mission@gip-recherche-justice.fr

Site Internet : www.gip-recherche-justice.fr

\section{Justice et nouvelles technologies}

Le texte qui suit est un guide de réflexion pour ceux qui, quelle que soit leur discipline, ont l'intention de répondre à l'appel à projets. II présente les orientations prioritaires de recherche retenues pour ce thème, dans le cadre desquelles une large part d'initiative est laissée aux chercheurs.

Nul n'est tenu de traiter de l'intégralité des axes de recherche proposés ci-après.

A ce texte sont joints deux documents, également disponibles sur le site de la Mission (rubrique "Présenter un projet") :

- une note rappelant les modalités de soumission des projets

- une fiche de renseignements administratifs et financiers, laquelle, dûment complétée, doit nécessairement accompagner toute réponse à l'appel à projets. 


\section{Justice et nouvelles technologies}

Cet appel à propositions s'intéresse à la place que les nouvelles technologies de l'information et de la communication (NTIC) occupent dans le processus de modernisation de la justice entendu comme un mouvement de réformes visant à introduire davantage de célérité, d'accessibilité et d'efficacité dans le traitement des affaires judiciaires. Il questionne aussi les effets directs et indirects, positifs ou considérés comme «pervers» que l'introduction de ces moyens technologiques a pu entraîner dans les pratiques judiciaires.

Les NTIC, nées de la convergence de l'informatique, des télécommunications et de l'audiovisuel regroupent un ensemble de matériels et de logiciels permettant de manipuler plus aisément une information dont les supports sont dématérialisés, de la convertir, la stocker, la gérer, la transmettre et la retrouver.

D'abord circonscrite à l'aide à la rédaction des actes (plutôt qu'à l'aide à la décision comme il était dit parfois) puis aux possibilités nouvelles que la dématérialisation permettait d'envisager quant à la conservation et à la circulation des documents, la question s'étend aujourd'hui au travail collaboratif, nombre de logiciels autorisant l'élaboration simultanée d'un acte par plusieurs auteurs et affranchissant les parties de l'obligation d'une présence physique en un même lieu et en un même temps.

Ces techniques et leurs mises en oeuvre suscitent chez les professionnels du droit et de la justice, qui en exploitent déjà certaines ressources (le réseau e-barreau ou quelques tribunaux de grande instance, par exemple), de grands espoirs en matière de rationalisation du travail en améliorant les pratiques et en créant des types nouveaux de solutions aux problèmes de gestion collective des connaissances (travail collaboratif). Mais elles engendrent aussi des craintes en matière de confidentialité et de sécurité des données et sont parfois soupçonnées de porter atteinte à l'égalité des citoyens - voire des professionnels du droit eux-mêmes -, et de remettre en cause certains principes fondamentaux du droit.

\section{Champs thématiques envisageables (liste purement indicative):}

- l'adaptation réciproque des pratiques judiciaires, règles de droit et procédures et des NTIC

- la formation des agents, la mise à disposition des moyens matériels adéquats

- l'incidence des NTIC sur le fonctionnement et l'organisation des tribunaux

- l'évolution des relations entre le milieu professionnel judiciaire et l'extérieur

Quatre niveaux peuvent, très schématiquement, être envisagés pour une analyse de la mise en œuvre des NTIC, des contraintes et exigences qu'elles supposent, des effets, souhaités ou non, qu'elles génèrent.

- la gestion de l'activité judiciaire : gestion de la juridiction elle même, gestion des dossiers. C'est le fonctionnement de la justice qui est concerné, dans sa dimension organisationnelle,

- la production judiciaire: la dématérialisation des actes modifie le processus d'élaboration des décisions. Cette dimension touche au procès, à sa nature (recours à des bases de données jurisprudentielles, numérisation des procédures pénales, informatisation de la mise en état des dossiers civils...). aux modes de relation avec les auxiliaires de justice...)

- les rapports entre la justice et les justiciables : ce point renvoie naturellement au rôle des avocats (par exemple dans le cas de plainte avec constitution de partie civile déposée par voie électronique par l'intermédiaire d'un avocat). Peut aussi être 
mentionnée la création d'un portail grand public d'accès à la justice qui permet d'obtenir, via Internet, tout un ensemble d'actes administratifs.

- l'activité des professions du droit et de la justice : autant que les magistrats et fonctionnaires travaillant dans les tribunaux et les cours - dont l'activité, la place dans la juridiction peuvent être transformées par le recours aux nouvelles technologies -, avocats, notaires, huissiers sont très directement concernés, la nature même de leur fonction pouvant notablement évoluer sous l'effet des NTIC.

N.B. Sont exclues du champ de cet appel d'offres les questions à caractère technique en rapport avec les moyens affectés à l'administration de la preuve (empreintes génétiques, interception de correspondances par voie de télécom- munication...), avec les modes d'exécution des sanctions (bracelet électronique...) ou encore avec les problèmes d'expertise posés par le traitement judiciaire des litiges impliquant les NTIC.

L'objet retenu est bien la relation justice/nouvelles technologies de l'information et de la communication.

Problématiques possibles (là encore, liste non exhaustive) :

- Le temps judiciaire : quid de la nécessaire maturation de la décision ?

- L'« acculturation » des agents (magistrats, greffiers, OPJ etc.) : comment s'opère l'acquisition des connaissances et quelles transformations exerce-t-elle sur les représentations?

- L'interactivité entre culture technique et culture judiciaire

- La transformation des chaînes de traitement

- L'évolution des rapports justiciables / institution, en lien avec la modernisation des moyens de l'administration

- La nature de l'assistance apportée au magistrat par les NTIC. La question se pose, par exemple, en matière de barèmes (objectivation de situations singulières) : que devient l'acte de juger s'il est encadré, voire contraint, par des procédures automatisées ? D'où le débat entre barème indicatif et barème impératif.

A partir de cette définition du périmètre d'intérêt et des suggestions de problématiques énoncées ci-dessus, nombreuses sont les questions que pose le rapport singulier de la justice et des nouvelles technologies. Singulier parce que la justice n'est pas tout à fait comparable à d'autres administrations. Ses missions sont spécifiques car étroitement liées au pouvoir - exercé au titre du monopole de la "violence légitime » - de prononcer des sanctions, notamment de privation de liberté. Elles supposent d'être étroitement encadrées par le droit, la procédure, la compétence et la probité de ceux qui les exercent. Dès lors, la place des nouvelles technologies dans cet univers extrêmement diversifié de règles et de pratiques, dans ce milieu dominé par la "culture papier », seule apte, jusqu'à il y a peu, à laisser des traces des procédures et des décisions, n'est pas toujours apparue comme naturelle.

L'analyse devrait aider à comprendre ce qui a fait qu'une nouvelle culture, technique, est venue se greffer sur des us anciens, voire se substituer à eux. Quels ont été les vecteurs, les acteurs de cette mutation, au sein et en dehors de la justice ? Comment s'articulent logiques techniques et logiques juridiques et judiciaires ? Les objectifs traditionnels associés à ces dernières peuvent-ils être transformés par les potentialités ou contraintes techniques ? Un nouveau sens peut-il ainsi être donné à l'action judiciaire?

Quel a été l'impact de ces dispositifs sociaux techniques, notamment sur les quatre « niveaux » proposés ci-dessus (gestion, production, relations, professions) ? Pour chacun d'entre eux devraient être analysées les recompositions qui ont pu se produire, par exemple au sein des services ou des professions, lesquels sont généralement fortement structurés.

Quels sont les bénéfices escomptés des nouvelles technologies, quelles en sont les limites, techniques, humaines, juridiques ? Ainsi, s'agissant par exemple de la visioconférence, désormais bien admise dans certains cas, elle n'est pas sans soulever de nombreuses 
questions, dès lors qu'elle est envisagée dans le contentieux de la détention (place de l'avocat, absence du détenu dans la salle d'audience...).

Quels en sont aussi les dangers : déshumanisation, standardisation de la justice, inégalités (entre justiciables, entre professionnels...), remise en cause de principes fondamentaux..., les difficultés : sécurité des réseaux...

Le risque existe-t-il d'un développement non contrôlé des affaires portées devant la justice, du fait d'une accessibilité accrue de celle-ci ? Une attention particulière pourrait, sur ce point, être portée non seulement sur la justice pénale ou la justice administrative, plus avancées, semble-t-il, que la justice civile en matière d'usage des nouvelles technologies, mais aussi sur ce qui est parfois appelé «les modes électroniques de règlement des litiges ", en référence aux modes alternatifs de règlement de ces mêmes litiges (médiation, mais aussi arbitrage...).

Plus fondamentalement, quelle est la marge entre le juge « assisté » par l'informatique et le juge « suppléé » par celle-ci ?

Si une telle question suppose une réponse globale, du fait de sa généralité, de sa transversalité, il est manifeste que la plupart des interrogations pointées ci avant nécessitent un traitement spécifique, prenant en compte la diversité tant des techniques elles-mêmes que des lieux ou situations de leur application : telle profession, telle juridiction, tel service, telle procédure, telle fonction...

II serait certainement très intéressant de comparer, ou du moins de rapprocher, l'institution judiciaire avec d'autres secteurs d'activité. Celui de la médecine, entre autres, se prêterait apparemment assez bien à cet exercice, l'usage de nouvelles technologies, notamment de diagnostic et d'intervention à distance ayant bousculé les pratiques traditionnelles.

Enfin, le mouvement de recours aux nouvelles technologies dans la justice apparaît, dans la plupart des pays, assez généralisé, ce qui pourrait donner tout son intérêt à une approche comparative. Ainsi, dans une note parue sur le site officiel de la présidence tchèque de I'Union européenne, le 16/ 12 / 2008, il est dit: "L'interconnexion des registres électroniques tenus par les Etats-membres, le recours à la visioconférence dans le cadre des procédures judiciaires transfrontalières ou la création d'un portail européen de justice en ligne (e-justice) pour un accès uniforme au droit pour tous les citoyens de l'UE et les spécialistes du droit : dans tous ces domaines, l'utilisation des nouvelles technologies de l'information peut rendre la pratique judiciaire bien plus efficace et apporter ainsi aux citoyens une véritable valeur ajoutée ». 